\title{
Predictive Factors for Successful Percutaneous Sclerotherapy of Venous and Lymphatic Malformations
}

\author{
Anchalee Churojana, MD \\ Laksanawadee Mahiwan, MD \\ Dittapong Songsaeng, MD \\ Rujimas Khumtong, MD \\ Saowanee Homsud \\ Diagnostic Division, Department of Radiology, Faculty of Medicine Siriraj Hospital, Mahidol University, Bangkok, Thailand
}

\begin{abstract}
Objectives: To determine the predictive factors for good response outcome of venous (VMs) and lymphatic malformations (LMs) by percutaneous sclerosing therapy and to compare the complications associated with bleomycin versus alcohol treatment.

Methods: A retrospective analysis of 225 patients with lymphatic and venous malformation who had treated by percutaneous sclerotherapy using alcohol and/or bleomycin was performed The treatment outcome was graded from $0-3$, in orderly of clinical responsiveness by using visual assessment of changing in size and subjective improvement of symptoms. Predictive factors of good treatment outcome were determined by uni- and multivariate analysis which were conducted on sex, age, onset of disease, location, type, characteristic of lesion and sclerosant usage.

Results: Of 225 patients, $87.6 \%$ were VMs and $12.4 \%$ were LMs. VMs were predominating in female (6.2:3.8). Between ethanol and bleomycin, there was no statistical significant of treatment sessions, dose and treatment outcome. $(p=.42)$ Sclerosing therapy provided better response on VMs than LMs. $(49.2 \%$ and $21.4 \%$ respectively, $\mathrm{p}=.002)$, and had more effective on focal lesions than diffuse patterns $(64.9 \%$ and $26.1 \%$ respectively $p<.001)$ The gender, onset of disease, locations, characteristic on venography and preceding treatment showed no correlation with treatment result. Major complications of ethanol occurred in $38.6 \%$,including hematuria, gangrene, facial paralysis, pulmonary embolism and death. Minor adverse reactions of bleomycin encountered in $19.8 \%$ with hyperpigmentation, flu-like symptom and localized fibrosis.

Conclusion: Both absolute alcohol and bleomycin were effective sclerosing agents for percutaneous treatment of venous malformations, but no serious complications encountered with bleomycin. The characteristic of focal single lesion, at any region of body part, can be a predictor for good response.
\end{abstract}




\section{Introduction}

Venous malformation (VM) and lymphatic malformation (LM) are benign congenital abnormalities. They grew proportionately with the patients and did not regress ${ }^{1}$. Although presented at birth, they may appear clinically at any age throughout the life. The presentations of VM and LM are variable including asymptomatic, swelling, pain, ulcerate, bleed, and cosmetic concerns. The clinical severity is related to their location and extension. If located within the oral cavity, these lesions may lead to dysphagia, difficulties with speech or swallowing, orthodontic concerns, and airway obstruction $^{1-3}$.

Treatment of VM and LM were difficult, compose of surgery, laser, steroid therapy, and sclerotherapy ${ }^{4}$. Surgical resection may be hazardous, leading to major blood loss, and incomplete resection. Recurrence and deformity were also common after surgical resection ${ }^{1}$. Laser therapy often had an extensive scar and may ineffective in some patient especially in deep seated lesion. Sclerotherapy, as an alternative treatment method, has proven its efficacy and now advocate as the treatment of choice ${ }^{1,3-5}$. A variety of sclerosing agents was described in the literature, including ethanol, detergents, hyperosmotic solutions, OK 432, ethibloc, and bleomycin ${ }^{2,3}$.

In our institute, percutaneous sclerosing therapy has been operated by using absolute alcohol and bleomycin as the sclerosing agents. With regard to difference of morphology or characteristic of the lesions and response of sclerosing therapy, the objective of this study was to evaluate the predictive factors for successful treatment outcome of venous and lymphatic malformations, and to compare the complications associated with bleomycin versus alcohol treatment.

\section{Material and methods}

Patients: A retrospective review of 258 patients with clinical diagnosis of venous and lymphatic malformations who had referred to our Interventional Neuroradiology division, Radiology department, Siriraj hospital, Mahidol university for sclerosing therapy between July 2001 and July 2011 was performed. An informed consent was given to the use of sclerosing therapy after patient's counseling. A data collection in each patient including age, sex, location of lesion, clinical presenting symptom, dose of sclerosant, number of treatment sessions, clinical response, side effects and follow-up. Colour photographs were taken before treatment and during every follow up period.

The diagnosis was confirmed during the operation by yielding the content either blood or lymphatic fluid aspiration directly from the lesion by percutaneous puncture.

The exclusion criterias were

1. Patients who refused treatment.

2. Coexistence to other types of malformation or syndromic disease.

3. The diagnosis could not be confirmed during operation.

4. The patients who had less than 6 months follow up after first sclerotherapy.

Of 258 patients, there were 225 patients who met our criteria and enrolled in this study.

The imaging studies were not routinely performed, but requested in cases where boundaries were difficult to evaluate or deep seated locations.

Technique and sclerosing agents: The procedure was performed under general anesthesia or 
regional block depending on site of each lesion and anesthesiologist's decision. Each lesion was assessed percutaneously by using a 20 to 22 gauze Teflon-sheathed needle canula puncture needle through a normal skin. Once venous blood or thin yellow fluid was freely aspirated, contrast medium was injected to evaluate the characteristic and to estimate the volume of the lesion.

The characteristic of the lesions were categorized to be

- Focal; if involvement of one region of the body such as finger, lip.

- Diffuse, if involvement of more than one region, such as hand and forearm, lip and tongue

- Isolate: if there were no venous outlet connecting to systemic circulation

- Systemic venous connection

Before June 2008, all VMs were treated solely by absolute alcohol while no percutaneous treatment for LMs, since then, bleomycin was the only agent using for both VM and LM.

For technique of percutaneous sclerosing injection, it was performed similarly, for both ethanol and bleomycin, under fluoroscopic control. Manual compression of venous outlet or venous pressure tourniquet was applied during injection, if systemic venous connection with the lesion was identified on test percutaneous venography, and this was maintained for at least 10 minute after finishing to retain the sclerosing agent and to minimize systemic contamination. Multiple punctures were required in the same session, if the lesion contained several noncommunicating cavities.

The absolute alcohol was opacified with nonionic contrast medium at the ratio of $3: 1$ by volume. It was slowly injected under fluoroscopic control. The amount of each injection was estimated from prior test injection and termination when near total lesion was opacified or spillage into venous outlet was visualized. The maximum amount of absolute alcohol was $1 \mathrm{ml} / \mathrm{kg}$ body weight but not exceed $40 \mathrm{ml}$ in each session ${ }^{6}$.

Prolonged intubation or prophylactic tracheostomy was required, if the lesion located around tongue or oral cavity.

Bleomycin was mixed at the dilution of $1 \mathrm{mg} /$ $\mathrm{ml}$ by normal saline. It was injected under roadmap fluoroscope after contrast test injection. Injecting bleomycin was stopped as soon as the contrast medium column reached the venous outlet. The amount of the injection varies according to the size of the lesion. In children under 1 year of age, the maximum dose per injection was limited to $1 \mathrm{mg} /$ $\mathrm{kg}$. Patients who were older than 1 year of age, a single dose of $15 \mathrm{mg}$ was never exceed ${ }^{7}$. For LM lesion, before starting bleomycin injection, the lymph fluid was aspirated as much as possible with the aim to minimize dilution effect of the sclerosant.

Repeated maneuver of sclerotherapy were obtained in 6-8 weeks for the large or the remaining lesions which were re-evaluated at 4-week follow up period.

The accumulation dose of bleomycin was collected in each patient, not to exceed $450 \mathrm{mg}^{7}$.

Outcome evaluation: The result of treatment was evaluated by comparison the size and clinical symptoms preceding treatment and those at the last visit. The size and morphology of the lesion was evaluated by visual assessment from photographs. The clinical responses were included degree of pain, bleeding and ulcer healing, using patient self-assessment, which were evaluated by direct interview with the patients or parents. 
Treatment outcome was evaluated by means of clinical response and quantified by grading of the consensus between Interventional Neuroradiologists and the patients or their parents into 4-point scale, as the followings

Grade $\mathbf{0}=$ stable or worse

Grade $1=<50 \%$ improvement of symptoms

Grade $2=50-75 \%$ improvement of symptoms

Grade $\mathbf{3}=>75 \%$ improvement of symptoms

The treatment outcome grade 2 and 3 were defined as good response.

Complications of the treatment by ethanol and bleomycin were recorded separately.

Statistical analysis: The factors which may associate with treatment outcome, including gender, period of disease-onset, site, type and characteristics of lesions, preceeding treatment and sclerosants were analyzed, by using chi-square test. The sclerosants were grouped according to period of using as ethanol alone, ethanol and bleomycin and bleomycin alone. The correlation of patient's age and treatment outcome was evaluated by the unpaired t-test. The correlation between treatment outcome and the predictive factors were determined by the multiple logistic regressions. $P<.05$ was considered statistically significant. Data analysis was conducted by using the PASW statistic 18.8 software.

\section{Result}

Of total 225 patients, there were $197 \mathrm{VMs}$ (87.6\%) and 28 LMs. (12.4\%). VMS were predominating in female (6.2:3.8). The mean age was 22.9 years, ranging from 5 months to 68 years. The presenting symptoms, locations and characteristics of the lesions were demonstrated on table 1. All pre- sented with mass whereas $11 \%$ of them had additional sequelae of the diseases including pain, bleeding, lymphatic leakage, and ulceration. The lesions were revealed since birth at $48 \%$ and later in life at $52 \%$. The locations of the diseases were found more common at extremities (61.33\%) than at head and neck region (35.11\%), and the other sites (3.6\%) were included shoulder, chest wall, back, abdominal wall and glan penis. Focal and diffuse involvements of the diseases were found rather equally (50.7\% and $49.3 \%$ respectively). Preceding therapy was documented in $35.6 \%$, including surgery, laser and steroid injection.

Concerning characteristic of venography, most lesions $(91.1 \%)$ had connection of venous drainages into systemic circulations either with or without venous ectasia, whereas $8.9 \%$ had isolated lesion.

Among groups of sclerosant using (ethanol only, ethanol and bleomycin, bleomycin only), there is no statistical significant of treatment sessions, dose and treatment outcome. $(p=.42)$

The average follow-up period was 13.5 months (range 6-90 months). The treatment outcome of those 225 patients were grade 0 at $7.6 \%$, grade 1 at $20 \%$, grade 2 at $26.7 \%$ and grade 3 at $45.8 \%$.

Among patients with grade 3 treatment outcome, VM had more response to sclerosing therapy than LM (49.2\% and $21.4 \%$ respectively $p=.002)$, and focal lesions had more effective than diffuse lesions (64.9\% and $26.1 \%$ respectively $p<.001)$. The gender, onset of disease, locations, characteristic on venography and preceding treatment showed no association with treatment result. The correlation between treatment outcome and the variable factors were demonstrated on table 4.

The VMs and focal lesions had been con- 


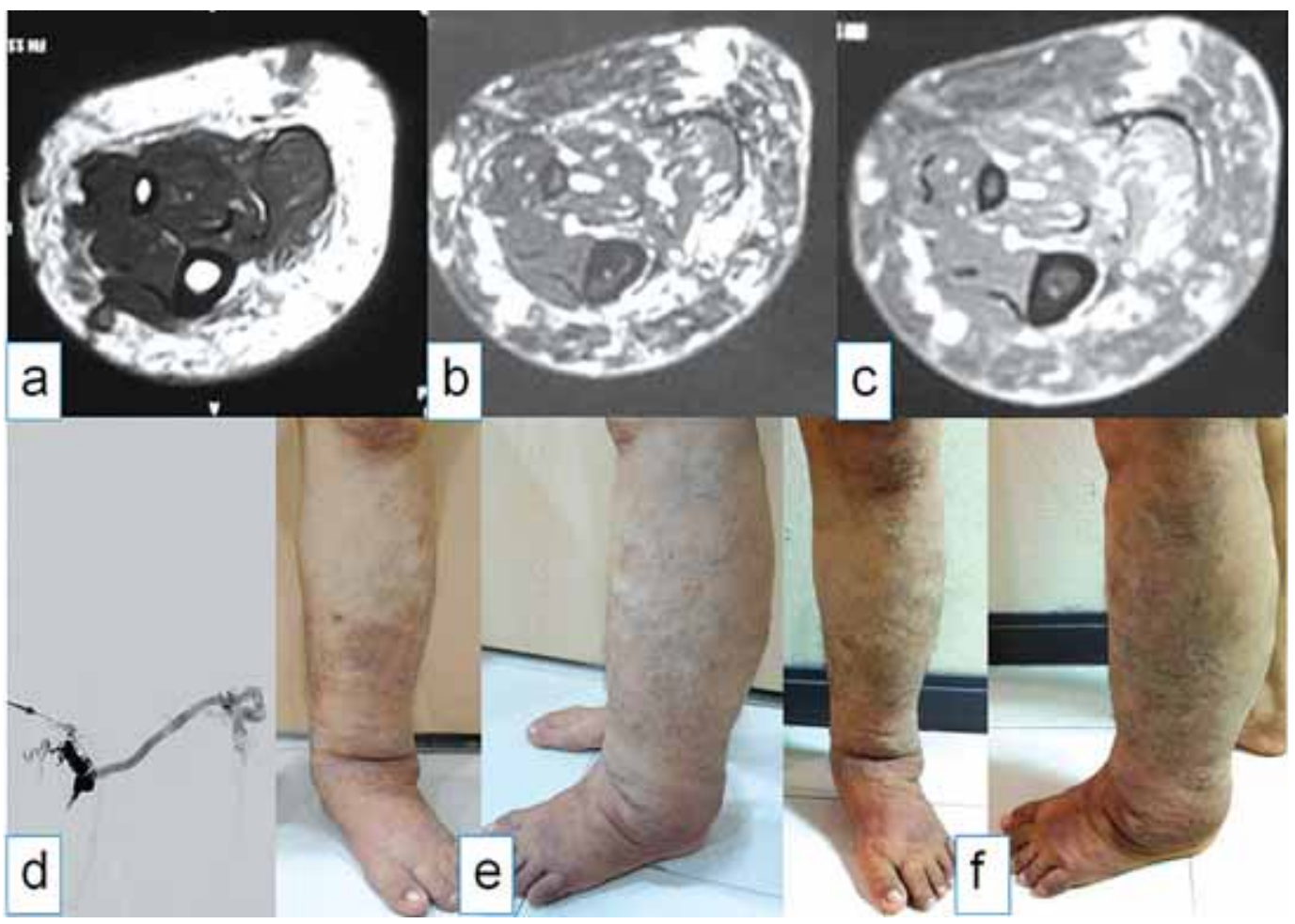

Fig. 1 VM with grade 0 treatment outcome; A 31-year-old male with asymptomatic diffuse type of VM involving left leg, ankle and foot had 3 sessions of bleomycin injection with accumulation dose at $45 \mathrm{mg}$. (a)T1WI, (b) T2WI/ SPAIR (c) T1WI/GD/FS MR imaging of the left leg revealed superficial diffuse venous malformation (d) Percutaneous venography (before sclerosant injection) showed abnormal dilated and tortuous venous structures with systemic venous connection.(e) pre-treatment photograph. (f) Photograph taken at 1 month after last session of sclerotherapy. Note that no significant difference in the appearance.

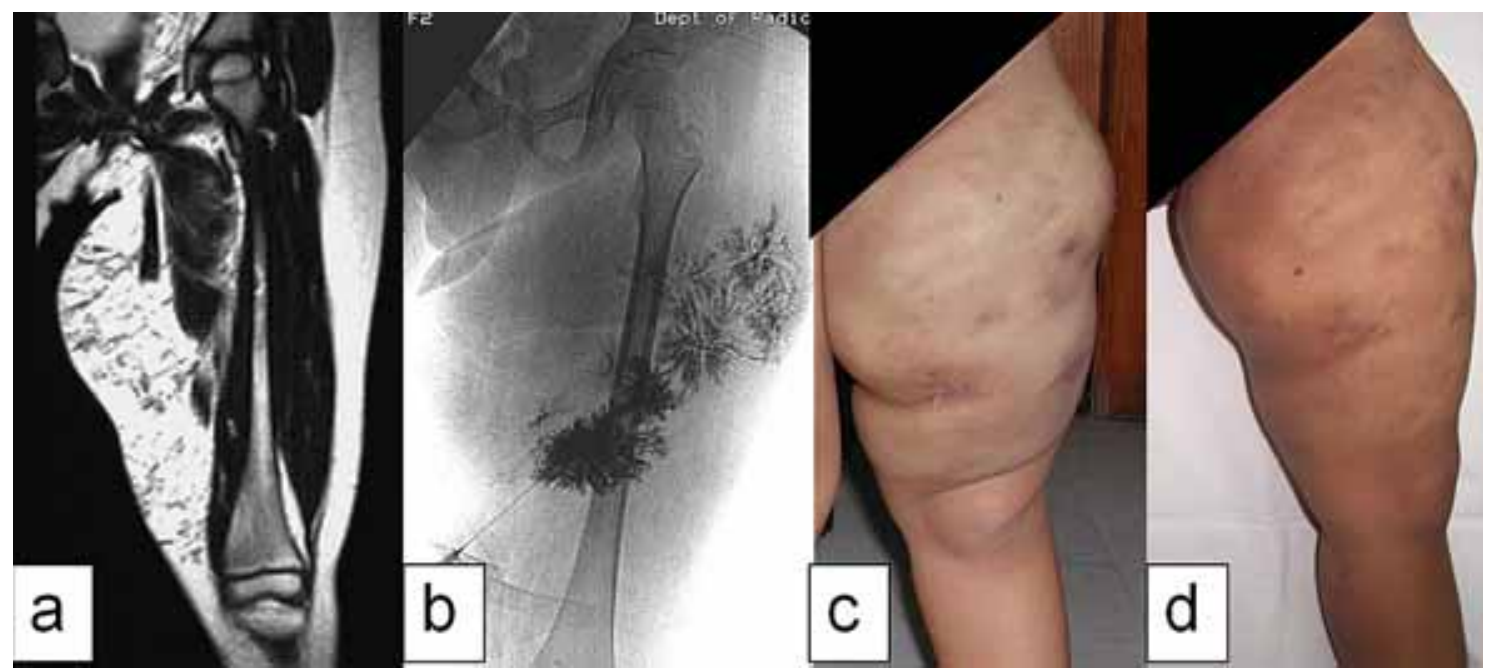

Fig. 2 VM with grade 1 treatment outcome; A 3-year-old girl with diffuse type of VM at left thigh and clinical of pain was treated by one session of $15 \mathrm{mg}$ bleomycin injection. T1WI MR imaging of left thigh revealed diffuse superficial venous malformation (a) that showed abnormal dilated venous structures without systemic venous connection on percutaneous venography - before sclerosant injection (b). The pre-treatment photograph (c) and photograph at 6 months after first session of sclerotherapy (d) show minimal improvement. However the clinical presentation as pain was resolved. 


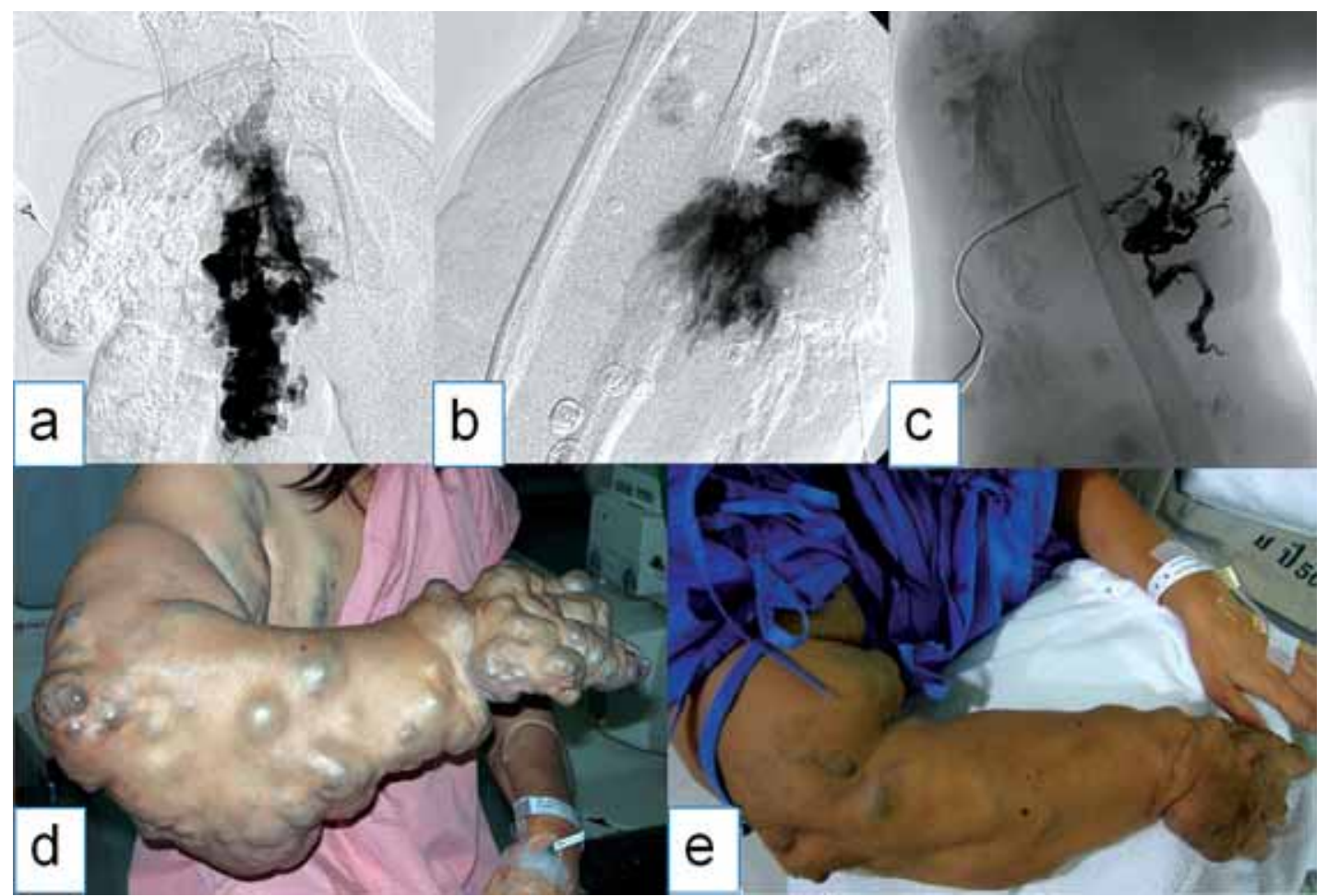

Fig. 3 VM with grade 2 treatment outcome; A 40-year-old female with cosmetic concerning VM at right hand, forearm, arm and anterior chest wall had 18 sessions of alcohol injection with total dose at $731 \mathrm{ml}$ and 15 sessions of bleomycin injection with total dose at $75 \mathrm{mg}$. Multiple time of percutaneous venography before sclerosant injection was performed at right hand (a), right forearm (b), and right arm (c) that showed abnormal dilated and tortuous venous structures with systemic venous connection. Pretreatment photograph (d) and photograph taken at 90 months after first treatment (e) showed good response of the disease. Even though still remained some residual malformation but patient had good satisfaction about cosmetic concern. 


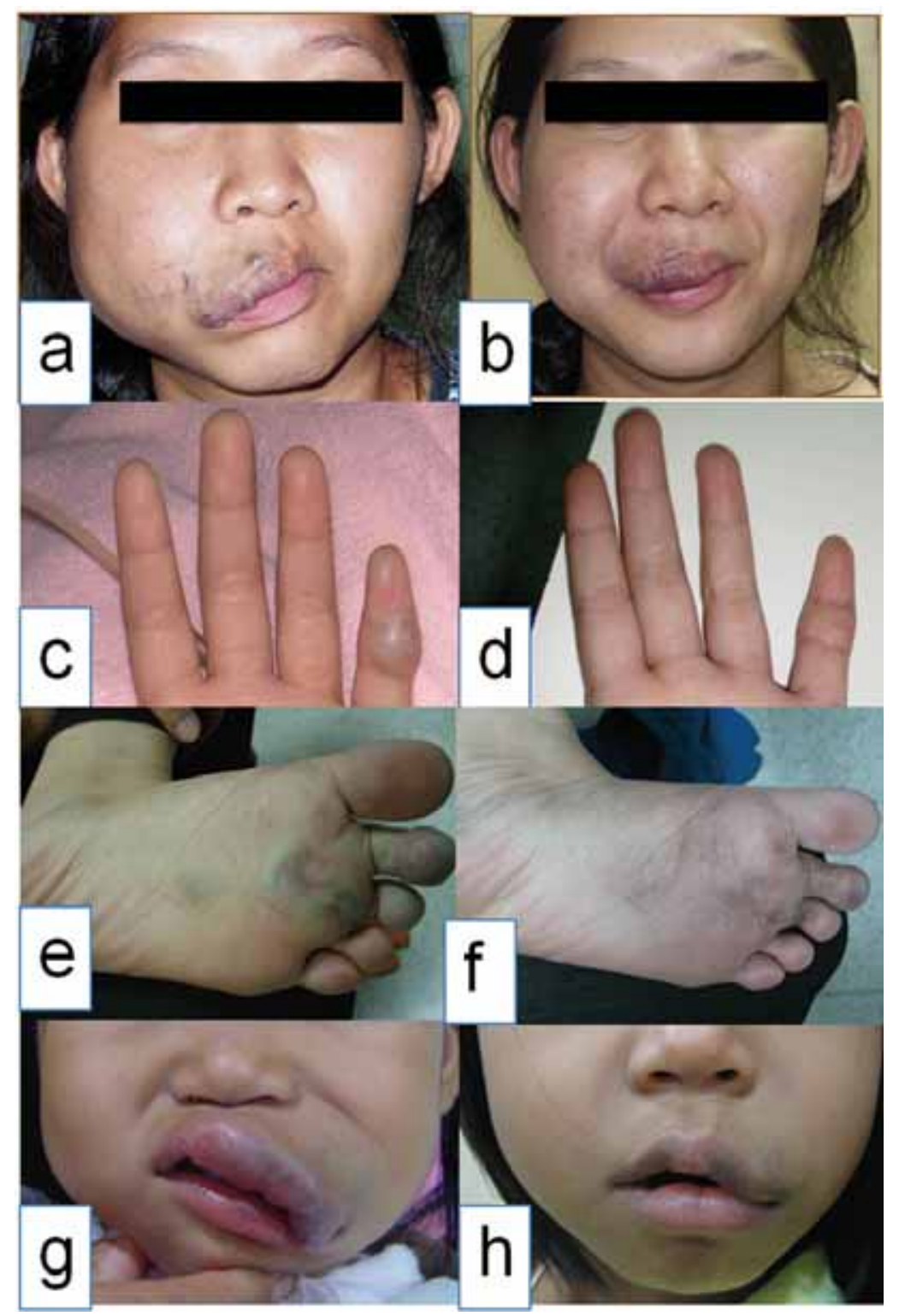

Fig. 4 VM with grade 3 treatment outcome; there are nearly obliteration of multiple sites of VM after percutaneous sclerotherapy, (a) pre-treatment photograph of a 30-year-old woman with VM at right lip and cheek, (b) photograph taken after 3 sessions of bleomycin sclerotherapy with total dose at $45 \mathrm{mg}$. (c) pre-treatment photograph of a 10-year-old girl with focal VM at left little finger, (d) photograph at 6 months after one session of sclerotherapy with $4 \mathrm{ml}$ of alcohol. (e) pre-treatment photograph of a 22-year-old man with focal VM at left foot, (f) photograph taken after 2 sessions of bleomycin sclerotherapy with total dose of $30 \mathrm{mg}$. (g) pretreatment photograph of a 5-year-old girl with focal VM at upper lip, (h) photograph taken at 27 months after 5 sessions of bleomycin sclerotherapy with total dose of $56.5 \mathrm{mg}$. 


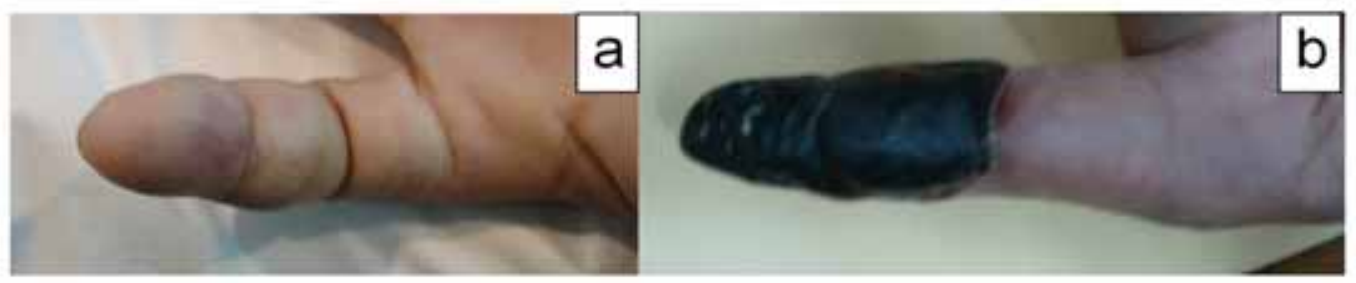

Fig. 5 Complication of alcohol sclerotherapy; a 31-year-old female with venous malformation at left 2nd finger and had previously surgery; pre-treatment photograph (a), she developed dry finger tip gangrene after one session of 4 $\mathrm{ml}$ alcohol injection (b). After 6 months follow up the lesion was not improved then she was consult for amputation.

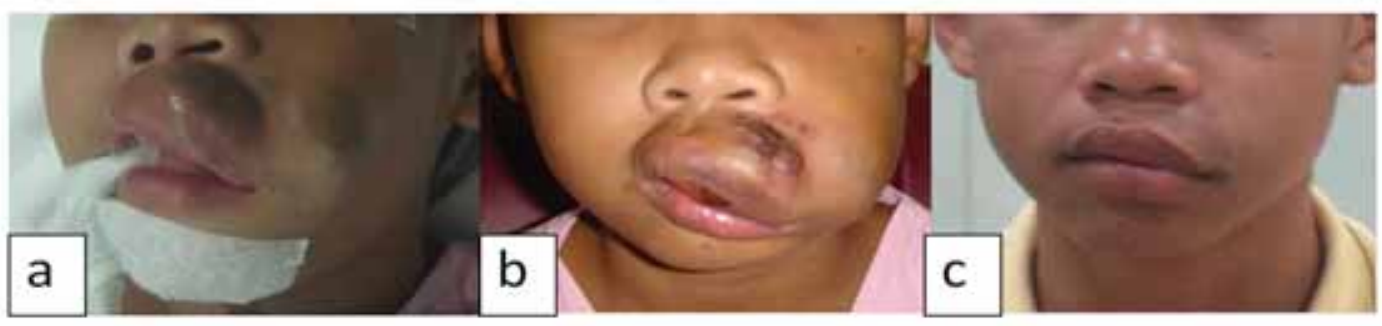

Fig. 6 Complication of alcohol sclerotherapy; a 13-year-old boy with VM at upper lip and left cheek, he had severe swelling at puncture site after $12 \mathrm{ml}$ of alcohol injection (a) and 5 days afterthat it showed minimal improvement (b). About 22 months after one session of alcohol injection and three sessions of bleomycin injection, near normal of the lesion was depicted.

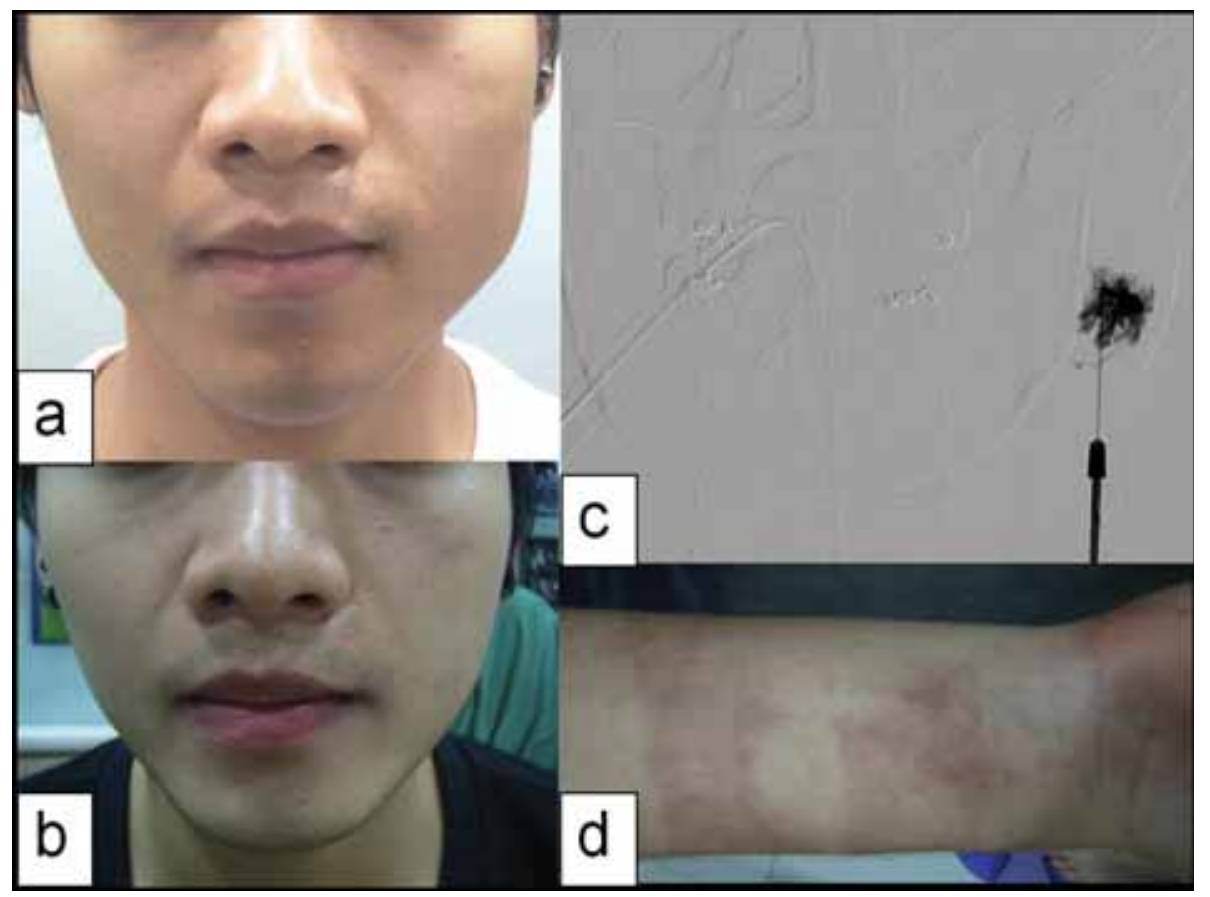

Fig. 7 Complication of bleomycin sclerotherapy; a 19-year-old male with VM at left cheek had one session of $15 \mathrm{mg}$ bleomycin sclerotherapy (a) pre-treatment photograph, (b) photograph taken at 7 months after first treatment the lesion was markedly regressed. The percutaneous venography revealed malformation without systemic venous drainage (c). He developed hyperpigmentation at right forearm which not improve after 6 months follow up, but the patient was accepting for this complication. 
Table1: Demographic and characters of venous and lymphatic malformations

\begin{tabular}{|c|c|c|}
\hline \multirow[t]{2}{*}{ Characteristics } & \multicolumn{2}{|c|}{ Malformation type } \\
\hline & LMs (28) & VMs (197) \\
\hline \multicolumn{3}{|l|}{ Sex (n, \% within group) } \\
\hline Male & $15,53.57 \%$ & $70,35.53 \%$ \\
\hline Female & $13,46.43 \%$ & $127,64.46 \%$ \\
\hline Mean age (yr) & 15.58 & 23.95 \\
\hline \multicolumn{3}{|l|}{ Presentation (n, \% within group) } \\
\hline Mass & $28,100 \%$ & $197,100 \%$ \\
\hline Bleeding & $2,7.14 \%$ & $3,1.52 \%$ \\
\hline Pain & $1,3.57 \%$ & $17,8.63 \%$ \\
\hline Ulceration & $0,0 \%$ & $1,0.51 \%$ \\
\hline Lymphatic leakage & $1,3.57 \%$ & $0,0 \%$ \\
\hline \multicolumn{3}{|l|}{ Previous treatment ( $\mathrm{n}, \%$ within group) } \\
\hline Laser & $1,3.57 \%$ & $3,1.52 \%$ \\
\hline Laser + surgery & $0,0 \%$ & $4,2.03 \%$ \\
\hline Surgery & $12,42.86 \%$ & $57,28.93 \%$ \\
\hline Steroid therapy & $1,3.57 \%$ & $2,1.02 \%$ \\
\hline \multicolumn{3}{|l|}{ Site (n, \% within group) } \\
\hline $\mathrm{H} \& \mathrm{~N}$ & $12,42.86 \%$ & $67,34.01 \%$ \\
\hline Upper extremities & $5,17.86 \%$ & $59,29.95 \%$ \\
\hline Lower extremities & $9,32.14 \%$ & $65,32.99 \%$ \\
\hline Other or multiple site & $2,7.14 \%$ & $6,3.05 \%$ \\
\hline \multicolumn{3}{|l|}{ Extension of lesion ( $\mathrm{n}, \%$ within group) } \\
\hline Focal & $12,42.86 \%$ & $102,51.78 \%$ \\
\hline Diffuse & $16,57.14 \%$ & $95,48.22 \%$ \\
\hline \multicolumn{3}{|l|}{ Morphology of lesion (n, \% within group) } \\
\hline Isolated without venous drainage & $5,17.86 \%$ & $15,7.61 \%$ \\
\hline Malformation with systemic venous connection & $23,82.14 \%$ & $182,92.39 \%$ \\
\hline
\end{tabular}

Table 2: Comparison of treatment sessions, dose and follow-up time among patient-groups treated with sclerosants

\begin{tabular}{|c|c|c|c|c|c|}
\hline \multirow{2}{*}{ Sclerosing agent } & \multicolumn{2}{|c|}{ No of sessions } & \multicolumn{2}{|c|}{ Dose } & \multirow[t]{2}{*}{$\mathrm{F} / \mathrm{U}(\mathrm{mo})$} \\
\hline & Alcohol & bleomycin & Alcohol (ml) & Bleomycin (mg) & \\
\hline \multicolumn{6}{|l|}{ Alcohol only (64) } \\
\hline Range & $1-19$ & - & $2-717$ & - & $6-74$ \\
\hline Mean & 3.27 & - & 80.5 & - & 14.78 \\
\hline \multicolumn{6}{|l|}{ Alcohol+bleomycin (24) } \\
\hline Range & $1-18$ & $1-8$ & $1.3-731$ & $0.6-120$ & $9-90$ \\
\hline Mean & 3.71 & 4.38 & 110.01 & 56.96 & 38.29 \\
\hline \multicolumn{6}{|l|}{ Bleomycin only (137) } \\
\hline Range & - & $1-10$ & - & $1-150$ & $6-70$ \\
\hline Mean & - & 2.26 & - & 28.27 & 10.09 \\
\hline
\end{tabular}


January-April 2013, Volume XIX No.I

Table 3: comparison of treatment outcome among patient-groups treated with sclerosants

\begin{tabular}{lcccc}
\hline \multirow{2}{*}{ Treatment outcome } & Alcohol & $\begin{array}{c}\text { Alcohol + } \\
\text { bleomycin }\end{array}$ & bleomycin & Total \\
\cline { 2 - 4 } & $6,9.4 \%$ & $0,0 \%$ & $11,8.0 \%$ & $17,7.6 \%$ \\
\hline Grade 0: (n, \% within group) & $9,14.1 \%$ & $4,16.7 \%$ & $32,23.4 \%$ & $45,20.0 \%$ \\
Grade 1: (n, \% within group) & $19,29.7 \%$ & $9,37.5 \%$ & $32,23.4$ & $60,26.7 \%$ \\
Grade 2: (n, \% within group) & $30,46.9 \%$ & $11,45.8 \%$ & $62,45.3 \%$ & $103,45.8 \%$ \\
Grade 3: (n, \% within group) & 64 & 24 & 137 & 225 \\
\hline Total $(\mathbf{n})$ & & & & \\
\hline
\end{tabular}

Table 4: correlation of variable factors and treatment outcome grading

\begin{tabular}{|c|c|c|c|c|c|}
\hline \multirow[b]{2}{*}{ Factors } & \multicolumn{4}{|c|}{ Outcome grading } & \multirow{2}{*}{$\begin{array}{c}\text { Total } \\
\mathbf{n}\end{array}$} \\
\hline & $\begin{array}{c}\text { Grade } 0 \\
n(\%)\end{array}$ & $\begin{array}{c}\text { Grade } 1 \\
\text { n (\%) }\end{array}$ & $\begin{array}{c}\text { Grade } 2 \\
\text { n (\%) }\end{array}$ & $\begin{array}{c}\text { Grade } 3 \\
n(\%)\end{array}$ & \\
\hline \multicolumn{6}{|l|}{ Gender, n (\% within group) } \\
\hline Male & $9(10.6 \%)$ & $18(21.2 \%)$ & $24(28.2 \%)$ & $34(40.0 \%)$ & 85 \\
\hline Female & $8(5.7 \%)$ & $27(19.3 \%)$ & $36(25.7 \%)$ & $69(49.3 \%)$ & 140 \\
\hline \multicolumn{6}{|l|}{ Onset, n (\% within group) } \\
\hline At birth & $7(6.5 \%)$ & $25(23.1 \%)$ & $35(32.4 \%)$ & $41(38.0 \%)$ & 108 \\
\hline Later & $10(8.5 \%)$ & $20(17.1 \%)$ & $25(21.4 \%)$ & $62(53.0 \%)$ & 117 \\
\hline \multicolumn{6}{|l|}{ Malformation type, $\mathrm{n}$ (\% within group) } \\
\hline VMs & $11(5.6 \%)$ & $36(18.3 \%)$ & $53(26.9 \%)$ & $97(49.2 \%)$ & 197 \\
\hline LMs & $6(21.5 \%)$ & $9(32.1 \%)$ & $7(25.0 \%)$ & $6(21.4 \%)$ & 28 \\
\hline \multicolumn{6}{|l|}{ Location, $\mathrm{n}$ (\% within group) } \\
\hline $\mathrm{H} \& \mathrm{~N}$ & $7(8.9 \%)$ & $11(13.9 \%)$ & $18(22.8 \%)$ & $43(54.4 \%)$ & 79 \\
\hline Upper extremities & $2(3.1 \%)$ & $12(18.8 \%)$ & $16(25.0 \%)$ & $34(53.1 \%)$ & 64 \\
\hline Lower extremities & $7(9.5 \%)$ & $19(25.7 \%)$ & $23(31.1 \%)$ & $25(33.8 \%)$ & 74 \\
\hline Other or multiple site & $1(12.5 \%)$ & $3(37.5 \%)$ & $3(37.5 \%)$ & $1(12.5 \%)$ & 8 \\
\hline \multicolumn{6}{|l|}{ Extension, $\mathrm{n}$ (\% within group) } \\
\hline Focal & $3(2.6 \%)$ & $13(11.4 \%)$ & $24(21.1 \%)$ & $74(64.9 \%)$ & 114 \\
\hline Diffuse & $14(12.6 \%)$ & $32(28.8 \%)$ & $36(32.4 \%)$ & $29(26.1 \%)$ & 111 \\
\hline \multicolumn{6}{|l|}{ Morphology, n (\% within group) } \\
\hline Isolated without venous drainage & $1(5.0 \%)$ & $1(5.0 \%)$ & $4(20.0 \%)$ & $14(70.0 \%)$ & 20 \\
\hline Malformation with systemic venous connection & $16(7.8 \%)$ & $44(21.5 \%) \backslash$ & $56(27.3 \%)$ & $89(43.4 \%)$ & 205 \\
\hline \multicolumn{6}{|l|}{ Previous treatment, $\mathrm{n}$ (\% within group) } \\
\hline Yes & $8(10.0 \%)$ & $16(20.0 \%)$ & $24(30.0 \%)$ & $32(40.0 \%)$ & 80 \\
\hline No & $9(6.2 \%)$ & $29(20.0 \%)$ & $36(24.8 \%)$ & $71(49.0 \%)$ & 145 \\
\hline
\end{tabular}


Table 5: Multivariate analysis of variables and good response of percutaneous sclerotherapy for venous and lymphatic malformations

\begin{tabular}{lccc}
\hline \multicolumn{1}{c}{ Variable } & P value & Odds ratio & $\begin{array}{c}\text { 95\% confidence } \\
\text { interval }\end{array}$ \\
\hline Sex; female & 0.271 & 0.716 & $0.395-1.299$ \\
Onset; at birth & 0.503 & 1.221 & $0.680-2.193$ \\
Malformation type; venous malformation & 0.001 & 3.682 & $1.635-8.290$ \\
Location; non-extremity & 0.108 & 0.545 & $0.268-1.108$ \\
Extension; focal & $<.001$ & 4.329 & $0.120-0.442$ \\
Morphology; Isolated without venous drainage & 0.066 & 0.269 & $0.060-1.193$ \\
Previous treatment; Yes & 0.542 & 0.829 & $0.453-1.517$ \\
\hline
\end{tabular}

firmed to be predictors for good response of sclerosing therapy by the multivariate analysis (table 5). The others were independent factor ( $p>$.05). The mean age of patients with and without good response were 22.8 and 23 years respectively which showed no statistical difference $(P=0.9)$.

The adverse reactions of bleomycin occurred in $19.8 \%$ including hyperpigmentation (either at the site of malformation or remote region) 25 patients (15.5\%), localized fibrosis $2(1.2 \%)$ and flu-like symptom 5 (3.1\%). However, no serious complication was recognized. In contrast to absolute alcohol, the major complications had happened in $21.8 \%$, including transient hematuria 10 (11.4\%), digital gangrene $1(1.1 \%)$, facial paralysis $1(1.1 \%)$, pulmonary embolism 1 (1.1\%) and decompensated right heart failure from increased pulmonary pressure with death $1(1.1 \%)$. In addition, swelling at puncture sites were found in nearly all cases, and skin blistering in 20 patients $(22.8 \%)$.

\section{Discussion}

Although both VMs and LMs are often asymptomatic, however, if there are accompanying pain and functional disturbance or cosmetic concern, treatment is required. The diseases can be found anywhere, in our series, peripheral locations were more common than head and neck region, which was rather different to other reports ${ }^{3,5,8}$. Even there is no predilection exists for either sex in VM and $\operatorname{LM}(5,9)$, but in our study VMs were more frequent in female (64.5\%).

Sclerosing therapy by percutaneous injection of liquid agents has been accepted to be the initial treatment of choice and has a high reported success rate ${ }^{1,10,11}$, with the aim to eradicate or reduce abnormal vessels by endothelial damage leading to inflammation, thrombosis and fibrosis with obliteration of the lesion finally ${ }^{2}$. Various sclerosing agents have been used including ethanol, ethibloc (radiopaque chemical sclerosant based on ethanol, corn protein, oleum papaveris, and propylene glycol), sodium tetradecil sulphate, polydocanol, ethanolamine oleate, and bleomycin ${ }^{1-3,10}$ In our experience, we used only absolute alcohol and bleomycin because of their availability, effectiveness and inexpensive.

Absolute alcohol induces cellular dehydration and endothelial wall protein denaturation resulting to subsequent permanent obliteraion of the vessel lumen $^{12}$. A $75 \%-95 \%$ response rate has been reported for ethanol sclerotherapy of $\mathrm{VM}^{2}$. However, 
injection of ethanol causes severe pain due to its local tissue reaction resulting in significant tissue swelling or even skin necrosis. Its severe complications can be extremely dangerous including tissue necrosis, permanent peripheral nerve injury, pulmonary hypertension and pulmonary embolism ${ }^{6}$. Similar to our population treated with alcohol, the major complications were recognized at a rate of $21.8 \%$, including hemolysis, gangrene, pulmonary embolism or even one death from uncompensated pulmonary hypertension.

Bleomycin is an antitumor agent, developed by Umezawa in 1966. Its mechanism is inhibition of DNA synthesis and has sclerosing effect on the endothelial cells $s^{4,7}$. First use in lymphangioma was reported successfully by Yura et al in 1977. Since that initial report, further studies have confirmed its utility in treating VMs. Thereafter, introduction of bleomycin in the treatment of venous malformations and hemangiomas was described by Muir et $\mathrm{al}^{7}$ in 2004, with response rate at $84 \%$ and minor complication were occurred total 8 in 95 cases including Flu-like symptoms, ulceration, cellulitis, and partial temporary hair loss. Since those reports, further studies have confirmed its utility as a sclerosing agent for lymphangiomas, hemangiomas and venous malformations with good results ${ }^{1,3-5,13}$. To date, no major complications with intralesional bleomycin injection was reported. Even the most concern, regarding the potential development of pulmonary fibrosis, which was dose dependent, found when administered during cancer therapy with accumulation dose higher than $300 \mathrm{mg}^{3.4}$, has never been described to its use as a sclerosing agent ${ }^{5}$. However, high incidence of residual disease associated with therapy using bleomycin alone was documented ${ }^{1.3}$.

At our institution, absolute alcohol was the only sclerosing agent of choice to treat VMs, until death occurred. In addition, many reports of intralesional bleomycin injection were described with a high success but less complication rates, we started using bleomycin first for LM in June 2008 and increased more in VMs. Until now, it completely replaced the use of ethanol injection. Our total 161 patients with bleomycin injection had the minor adverse reactions occurring in $19.8 \%$ with lesser postprocedural swelling, as compared to ethanol. Our experience was similar to those described in the literatures ${ }^{2}$.

Currently, regardless complications, between ethanol and alcohol, there is no consensus to the better sclerosant. Although there were reports of more efficacy of ethanol than bleomycin in the aspects of higher success rate and require fewer treatment sessions ${ }^{2}$, but the comparison analysis among groups of patients using sclerosant in our study; ethanol alone, ethanol and bleomycin and bleomycin alone, showed no statistical significant in sessions and outcome of treatment. Of our 225 patients treated with sclerosing therapy, satisfactory treatment outcome or good response, (grade 2 , 3) was accounted for $72.5 \%$

In this study, we postulated that focal lesions and venous malformations were the predictors of good treatment outcome. This was not surprised since a focal lesion involving only single body part was relatively smaller lesion with lesser extensive, thus it needed fewer session of treatment. In addition, this study was cross sectional study at one point of time, so it was not yet the final outcome of a number of patients with large and diffuse lesions who were still in the treating course.

To our experience, only $46.4 \%$ of LMs had good response to bleomycin injection which was 
not comparable to the previously published $|\mathrm{B}|$ series $^{4,5,14}$. However, our study involved a relatively small group of LMs (28 patients or $12.4 \%$ ), which was not sufficient for analysis and it needs further study. Nevertheless, on our observation, most lesions were multiple microcysts, with variable in sizes, and we felt that the larger the cystic space, the better response was.

Although our data seemed to have better outcome of treated isolated VMs than lesions with drainage of venous outlet (grade 3 outcome was 70\% and $43.4 \%$ respectively) but it showed no statistical significant $(P=.12$ in uni-variate analysis and .066 in multivariate analysis). As such, it was probably due to too different numbers of cases between the two groups ( $n=20$ and 205 respectively). Puig et a $1^{10}$ found that VMs with drainage into dysplastic veins and venous ectasia were more difficult to treat with sclerosing agents because of extensive drainage into the normal venous circulation and increased risk of embolic complications. As we performed pressure over venous outlet during injection, it might overcome this problem.

Yun et $\mathrm{al}^{12}$ reported female gender and not extremity location were significant associated with good response to percutaneous sclerotherapy. This was different from our study. We found no association between age, gender and location of the lesions with good response. However, in our study, the lesions at head and neck region had the highest success rate of sclerosing therapy.

The limitations of this study were retrospective design with a lack of standardized assessment tool, such as imaging study, to compare the result of treatment. The visual assessment on patients' photograph and patient's interview may be not enough statistical reliable.

\section{Conclusion}

Absolute alcohol and bleomycin were proved to be the effective sclerosing agents for percutaneous treatment of venous malformations. Although no significant statistical difference between their degree of success rate, but bleomycin showed superior patient's outcome due to absence of serious complications which could ensure satisfaction for both patients and physicians. Moreover, inferring from our study that the characteristic of focal single lesion, at any region of body part, can be a predictor for good response, and bleomycin provides more successful treatment outcome on VMs than LMs.

\section{References}

1. Jin Y, Lin X, Li W, Hu X, Ma G, Wang W. Sclerotherapy after embolization of draining vein: a safe treatment method for venous malformations. J Vasc Surg. 2008 Jun;47(6): 1292-9.

2. Spence J, Krings T, terBrugge KG, da Costa LB, Agid R. Percutaneous sclerotherapy for facial venous malformations: subjective clinical and objective MR imaging follow-up results. AJNR Am J Neuroradiol. 2010 May;31(5): 955-60.

3. Mathur NN, Rana I, Bothra R, Dhawan R, Kathuria G, Pradhan T. Bleomycin sclerotherapy in congenital lymphatic and vascular malformations of head and neck. Int J Pediatr Otorhinolaryngol. 2005 Jan;69(1):75-80.

4. Sung MW, Chang SO, Choi JH, Kim JY. Bleomycin sclerotherapy in patients with congenital lymphatic malformation in the head and neck. Am J Otolaryngol. 1995 Jul-Aug;16(4):236-41.

5. Rozman Z, Thambidorai R, Zaleha A, Zakaria Z, Zulfiqar M. Lymphangioma: Is intralesional bleomycin sclerotherapy effective? Biomed Imaging Interv J. 2011 Jul;7(3):e18.

6. Mason KP, Michna E, Zurakowski D, Koka BV, Burrows PE. Serum ethanol levels in children and adults after ethanol embolization or sclerotherapy for vascular anomalies. Radiology. 2000 Oct;217(1):127-32. 
January-April 2013, Volume XIX No.I

7. Muir T, Kirsten M, Fourie P, Dippenaar N, Ionescu GO. Intralesional bleomycin injection (IBI) treatment for haemangiomas and congenital vascular malformations. Pediatr Surg Int. 2004 Jan;19(12):766-73.

8. Jin Y, Lin X, Chen H, Li W, Hu X, Ma G, et al. Craniofacial venous malformations: magnetic resonance imaging features that predict treatment outcome. J Oral Maxillofac Surg. 2009 Nov;67(11):2388-96.

9. Som PM. Head and neck imaging. $4^{\text {th }}$ ed. St. Louis.: Mosby.; 2003.

10. Puig S, Aref H, Chigot V, Bonin B, Brunelle F. Classification of venous malformations in children and implications for sclerotherapy. Pediatr Radiol. 2003 Feb;33(2):99-103.

11. Lee KB, Kim DI, Oh SK, Do YS, Kim KH, Kim YW. Inci- dence of soft tissue injury and neuropathy after embolo/ sclerotherapy for congenital vascular malformation. J Vasc Surg. 2008 Nov;48(5):1286-91.

12. Yun WS, Kim YW, Lee KB, Kim DI, Park KB, Kim KH, et al. Predictors of response to percutaneous ethanol sclerotherapy (PES) in patients with venous malformations: analysis of patient self-assessment and imaging. J Vasc Surg. 2009 Sep;50(3):581-9, 9 e1.

13. Kullendorff CM. Efficacy of bleomycin treatment for symptomatic hemangiomas in children. Pediatr Surg Int. 1997 Sep;12(7):526-8.

14. Acevedo JL, Shah RK, Brietzke SE. Nonsurgical therapies for lymphangiomas: a systematic review. Otolaryngol Head Neck Surg. 2008 Apr;138(4):418-24. 